\title{
"Prendi la chitarra e vai / Se ti fermi invecchierai". Quando l'Italia era un paese di giovani e per giovani
}

\author{
di Cesare Grazioli
}

Ago 27, 2019 | La colonna sonora di una generazione $|\underline{0}|$

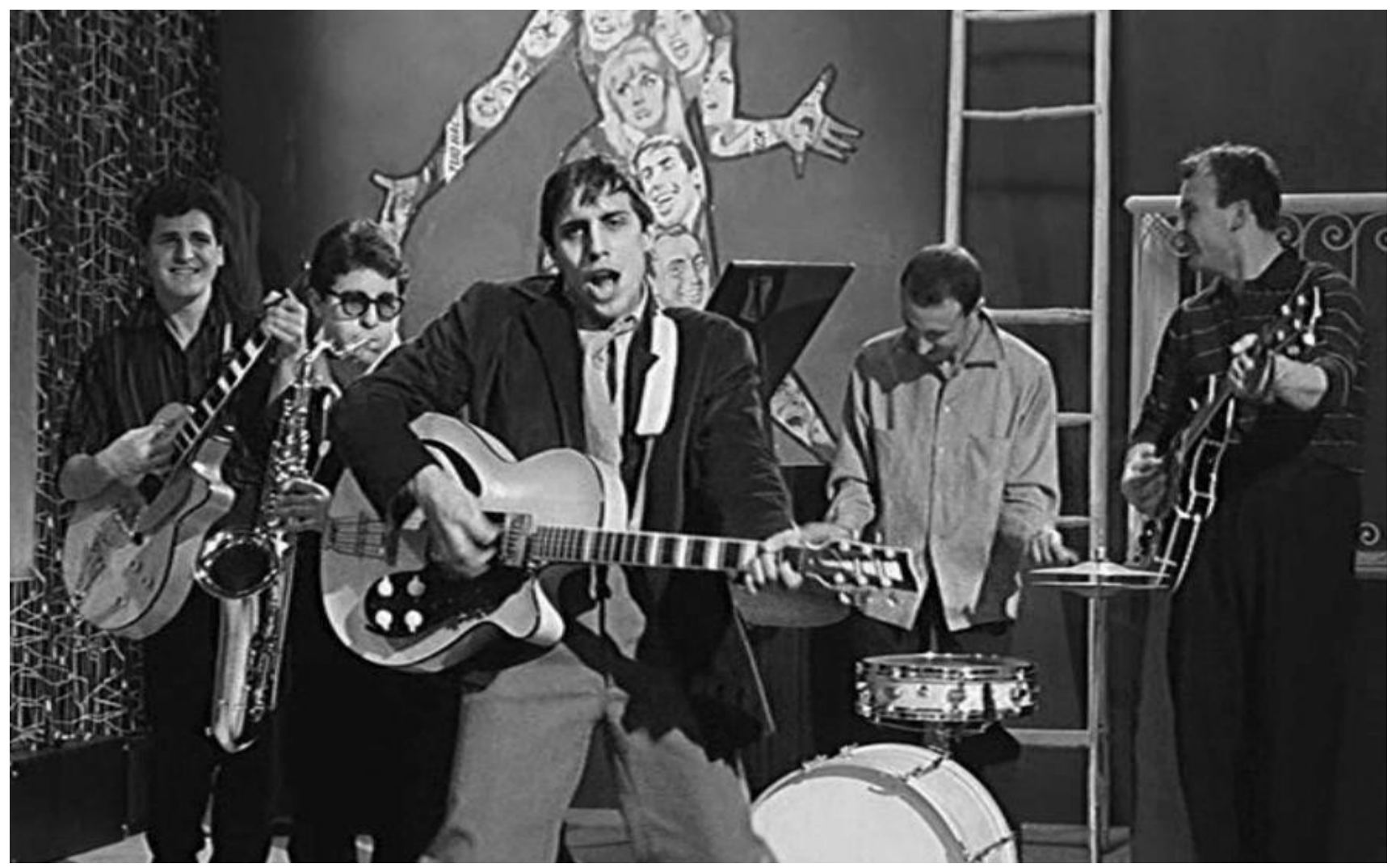

I Ribelli nel 1961. Al centro con la chitarra, Adriano Celentano. Foto nel pubblico dominio, Collegamento

\begin{abstract}
Gli anni Sessanta, a partire dal 1958 (anno d'inizio del "miracolo economico"), cambiarono radicalmente il panorama della società italiana, non solo sul piano economico, ma anche della cultura di massa, di cui la musica popolare è un aspetto e un indizio significativo. Sulla scia delle nuove mode musicali importate dall'America ma rielaborate in modo originale, i giovanissimi fecero irruzione nel mondo della canzone, sia come consumatori di musica (con un'esplosione del mercato discografico) sia come interpreti. Nacquero allora i giovani come fascia separata dal mondo degli adulti, un fenomeno sociale e antropologico senza precedenti. Ne fu rivoluzionata la tradizione melodica della "canzone all'italiana", che peraltro seppe raccogliere la sfida, e si moltiplicarono i filoni e le mode musicali.
\end{abstract}


Il testo che segue è costruito per gli studenti (in particolare quelli del triennio di scuola secondaria superiore), fornito loro direttamente o spiegato dal docente - comunque, con la visione diretta di un certo numero tra i video in esso proposti, tratti da YouTube.

L'obiettivo è di fare entrare nell'atmosfera degli anni Sessanta, da una prospettiva - la musica popolare, in Italia chiamata "leggera", insomma "le canzonette" - che si suppone coinvolga emotivamente gli studenti, susciti empatia. Che la storia insegnata non debba essere solo quella degli eventi politici, e neppure solo quella delle grandi tragedie collettive, dalle guerre ai genocidi, a parole è condiviso da ogni insegnante di buon senso: però il praticarlo nei fatti non è così ovvio e scontato.

\section{Come utilizzare il testo in classe?}

Per quanto riguarda i collegamenti con il programma di storia (discorso che ovviamente riguarda solo la classe quinta), tutto dipende dalle due opposte scelte possibili per il docente (entrambe legittime): se svolgerlo dopo avere affrontato gli anni Sessanta, come un approfondimento, e in tal caso cercare di fare ricavare agli studenti tali collegamenti; oppure se svolgerlo prima, come uno stimolo iniziale prima di affrontare sul libro di testo la storia di quegli anni. Come "stimolo iniziale", per entrare in modo "accattivante" nel periodo in questione, il testo si presta a uno svolgimento molto agile: una lezione frontale, o la lettura diretta del testo stesso da parte degli studenti, con il supporto del video-ascolto di un numero variabile di canzoni, e con le domande che il docente riterrà più opportune per sollecitare una restituzione collettiva.

Come approfondimento, il testo può servire a costruire un laboratorio (in questo caso, con fonti multimediali, appunto le canzoni) o uno "studio di caso", secondo la metodologia ampiamente sperimentata nei seminari estivi degli scorsi anni dell'Insmli, e largamente documentata su questa rivista. Con quale itinerario didattico?

Credo se ne possano ipotizzare diversi, e mi limiterò indicare, per sommi casi, quelli che sono venuti in mente a me.

\section{Quattro percorsi}

1. Una possibilità è quella di fare "spremere" agli studenti le differenze di clima culturale tra gli anni Cinquanta e i Sessanta, ricavabili dalle canzoni ascoltate (le 5-6 qui indicate per i Cinquanta, e almeno una dozzina per gli anni Sessanta); e di collegare poi queste differenze a quelle ricavabili dallo studio dei due decenni. Su questo, mi permetto di rinviare alla macro-periodizzazione sulla storia italiana dal dopoguerra al presente proposta all'inizio dell'articolo, che evidenzia come all'inizio degli anni Sessanta (nel 1958, se si vuole un anno) si ebbe la svolta forse più rilevante nella storia del nostro paese: l'inizio della modernizzazione, della trasformazione dell'Italia in una società industriale urbana, sia pure molto eterogenea per le sue profonde differenze territoriali.

2. Un percorso diverso, se si vuole più ludico ma che induce comunque gli studenti a cercare, pensare, ragionare e confrontarsi, con la modalità del lavoro di gruppo, potrebbe essere quello di fare costruire, a gruppi, una ipotetica Hit Parade degli anni Sessanta, con l'avvertenza però di includervi canzoni rappresentative dei molti filoni che si svilupparono e si intrecciarono in quel decennio, cioè (ma il seguente elenco potrebbe essere fornito dal docente, oppure fatto ricavare direttamente agli studenti dalla lettura del testo): canzoni "da spiaggia"; canzoni ballabili; canzoni di teen-idols; canzoni incentrate sull'essere giovani; canzoni di "urlatori" e canzoni melodiche; canzoni di stranieri che cantavano in italiano; canzoni di "complessi"; canzoni di cantautori; canzoni partecipanti a concorsi-festival canori. Se c'è tempo, si potrebbe poi fare confrontare in classe le "classifiche" costruite dai diversi gruppi tra i quali la classe si è divisa. 
3. Un altro percorso è interdisciplinare con Italiano e incentrato sulla "scrittura creativa": dopo avere letto e ascoltato i brani musicali sul tema dell'essere giovani negli anni Sessanta, si potrebbe chiedere agli studenti di cimentarsi nel comporre una canzone (solo il testo, o a discrezione anche con musica) sul tema dell'essere giovane oggi, nel 2019.

4. Infine, un possibile itinerario molto diverso da quelli finora indicati, viene suggerito dal sottotitolo di questo articolo: "Quando l'Italia era un paese di giovani e per giovani". Esso rimanda a un implicito ma bruciante confronto-contrasto con il presente di un'Italia che, almeno dall'inizio di questo secolo, non è un paese di giovani e per giovani, e sembra esserlo sempre di meno. Il punto di partenza di questa riflessione sarebbero ovviamente i dati demografici forniti in tabella, ma a questi dati altri se ne potrebbero aggiungere, da parte del docente o della classe sollecitata a cercarli. Qui mi limito a indicare gli aspetti più significativi del problema: l'altissima percentuale di disoccupazione giovanile in Italia, soprattutto ma non solo nel Sud; le pessime prospettive lavorative nei campi della ricerca e la conseguente tragedia della "fuga dei cervelli" dal nostro paese, che depaupera il nostro capitale umano delle migliori risorse, "regalandole" ai paesi che assorbono i nostri "cervelli in fuga". Se poi si vuole completare questa pista di riflessione, si dovrebbe mettere a fuoco il paradosso di un paese indotto oggi a considerare come suo principale problema l'immigrazione (che i dati statistici evidenziano al contrario essere ben poco significativa); mentre si dimentica o si ignora che il vero dramma italiano è oggi e nel prossimo futuro quello dell'emigrazione soprattutto intellettuale, e ad essa collegato quello del nostro declino sia demografico che intellettuale. Al fine di evitare che questo percorso di riflessione abbia un effetto troppo "depressogeno" per gli studenti, potrebbe essere impostato in positivo, a partire dalla domanda: "Come l'Italia potrebbe tornare (o: che cosa dovrebbe fare per tornare) ad essere un paese di giovani e per giovani?"

\section{Una periodizzazione per collocare storicamente gli anni Sessanta}

Le canzoni popolari sono un aspetto importante della cultura di massa: in un certo senso rappresentano la colonna sonora di una società, cosicché possono aiutarci a capire un determinato periodo storico. Ovviamente il rapporto è vicendevole: le canzoni ci dicono qualcosa di un periodo storico, il quale a sua volta ci aiuta a capire quelle.

Qui ci occuperemo delle canzoni degli anni Sessanta, e lo faremo a partire dalle domande: come vanno collocati storicamente gli anni Sessanta? quali aspetti li caratterizzarono?

Se abbracciamo la storia della società italiana dal secondo dopoguerra ai nostri giorni, in questi tre quarti di secolo è utile distinguere tre epoche, tre "mondi" molto diversi fra loro dai punti di vista sociale, economico, antropologico e della mentalità collettiva.

\section{Le tre fasi}

1. Prima ci fu un "lungo dopoguerra", che durò fino alla seconda metà degli anni Cinquanta, quando la società italiana era ancora prevalentemente agraria e rurale, con larghe sacche di povertà, in gran parte dialettofona e illetterata, radiofonica (cioè pre-televisiva) per quanto riguarda la forma più diffusa di comunicazione di massa; e si spostava a piedi e in bicicletta, con rari scooter e ancora più rare automobili.

2. Seguì il ventennio dalla fine degli anni Cinquanta alla fine dei Settanta, che fu segnato dalle trasformazioni più profonde e diffuse di tutta la nostra storia: l'affermazione di una moderna società industriale urbana, trainata dallo sviluppo dell'industria fordista, produttrice di beni di consumo di massa, in particolare automobili ed elettrodomestici; le forti migrazioni interne dalle campagne alle città e dal Sud al Nord; le profonde ridefinizioni dei rapporti 
sociali e di quelli di genere e di generazione, attuate in gran parte nel fuoco di aspri conflitti; il graduale innalzamento del livello di scolarizzazione e di alfabetizzazione; dal punto di vista delle forme di comunicazione di massa, l'avvento della cosiddetta "paleo-televisione" (in bianco-nero, in regime di monopolio pubblico, con forti valenze pedagogiche, secondo la sua triplice mission: "informare, istruire, intrattenere").

3. Negli anni Ottanta iniziarono a manifestarsi i tratti della società in cui viviamo tuttora, cioè del mondo attuale: il relativo declino del lavoro industriale a vantaggio del terziario e dei servizi, con il prevalere della finanza e della cosiddetta "economia di carta" della società post-industriale; l'emergere di nuovi settori strategici quali informatica, microelettronica e telecomunicazioni, biotecnologie, in vari modi collegati ai flussi di informazioni, e con le nuove forme di comunicazione in cui siamo immersi: una "neo-televisione" del tutto diversa da quella del ventennio precedente, internet, la telefonia mobile, i social network con la dilatazione del virtuale.

Sempre dagli anni Ottanta si sono avuti anche importanti mutamenti demografici: prima la cosiddetta "crescita 0 " e la fine dell'emigrazione (che aveva accompagnato le due fasi precedenti), poi il declino demografico, con un forte invecchiamento della popolazione, solo in parte compensato dai flussi di immigrazione; e più recentemente la ripresa dell'emigrazione, soprattutto nella forma, molto grave, della "fuga dei cervelli".

\section{La svolta del 1958}

Tratteggiare, anche solo sommariamente, questi "tre mondi" serve a evidenziare che gli anni Sessanta da una parte appartengono a un passato molto diverso dal presente; dall'altra parte, che essi segnarono una svolta molto marcata, l'inizio di "un altro mondo" rispetto al decennio immediatamente precedente.

Inoltre, nonostante gli anni Sessanta e Settanta vadano considerati dentro allo stesso "mondo", le differenze interne tra quei due decenni, e l'estrema poliedricità (o contraddittorietà) dei Settanta rende impossibile, in questa sede, occuparci di tutto il ventennio, e ci induce a concentrarci, appunto, sulla sua prima parte.

Se poi vogliamo mettere a fuoco un anno di svolta che inaugurò gli anni Sessanta, questo va certamente identificato nel 1958: 1'anno di entrata in vigore del Mercato Comune Europeo (dopo il trattato di Roma siglato l'anno prima), che divenne il principale destinatario delle nostre esportazioni; l'anno che inaugurò il quinquennio del "miracolo economico", con un'eccezionale crescita del Pil superiore al 6\% annuo; l'anno in cui gli occupati nell'industria sorpassavano quelli in agricoltura; l'anno in cui fu inaugurato il primo troncone dell'autostrada del Sole (appena un anno prima, era stato anche inaugurato il primo "Supermarket", anche se l'evento, gravido di conseguenze, passò all'epoca quasi inosservato). Come ora vedremo, però, Il 1958 va considerato un anno di svolta anche nella storia della canzone.

\section{Uno sguardo all'indietro: il panorama della canzone degli anni Cinquanta}

In Italia, tutto il dopoguerra musicale era stato all'insegna della più assoluta continuità con la tradizione della "canzone all'italiana", così come questa si era definita tra gli anni venti e trenta: melodica, sentimentale, con testi scritti in una lingua alta, aulica, molto lontana dal parlato; con temi spesso patetici e strappalacrime come amori infelici (quasi sempre per colpa di donne "tentatrici" e "peccaminose"), o la nostalgia del "bel tempo che fu", nonché i valori tradizionali della triade Dio-patria-famiglia. Vi era peraltro anche la variante delle canzonette molto allegre e orecchiabili, a volte filastrocche comiche o surreali, giocate sul nonsense o sui doppi sensi. In entrambe le varianti, erano quasi sempre canzoni non solo da ascoltare, ma anche da cantare e da 
ballare.

Già negli anni Trenta, poi ancora nei Cinquanta, si era creata una contrapposizione tra questa tradizione della "canzone all'italiana" e i ritmi e le musiche provenienti dall'America: il jazz e lo swing, poi anche il boogie woogie. Però tutto il decennio 1948-58 fu all'insegna della restaurazione dei valori tradizionali, nella società e nella politica (erano gli anni del centrismo, dopo la schiacciante vittoria elettorale della DC), così come nella musica. Come era accaduto durante il ventennio fascista, il potere politico (di fatto, la Democrazia cristiana) manteneva un controllo molto stretto sulla musica. In tutto il "lungo dopoguerra", infatti, la canzone rimase "di Stato", con il monopolio della radiofonia pubblica (l'Eiar, diventata Rai) e poi della televisione, dalla sua nascita nel 1954, e la sua controllata discografica, la Cetra.

\section{Perché Sanremo è Sanremo}

Il festival di Sanremo divenne il "tempio" della tradizionale "canzone all'italiana". Nato nel 1951, inizialmente sottotono con tre soli cantanti in competizione, divenne presto molto popolare grazie alla sua trasmissione via radio prima, poi in TV. I cantanti di fama erano non più di una dozzina, molto pochi anche perché non esisteva il legame cantante-canzone, che iniziò negli anni ' 60 , per cui lo stesso interprete ne eseguiva diverse, anche nella stessa competizione. La "regina della canzone" di tutto il decennio fu la bolognese Nilla Pizzi, che trionfò nelle prime due edizioni con Grazie dei fiori e poi con Vola colomba [www.youtube.com/watch?v=0vj26bTTl-Y].

Per avere un'idea della canzone all'italiana di quel decennio, basta ascoltare questi successi e altri come Campanaro [www.youtube.com/watch?v=6ttOFbCj83A], Vecchio scarpone

[www.youtube.com/watch?v=VvWH-jiwp2k], Papaveri e papere

[www.youtube.com/watch? $\mathrm{v}=\mathrm{I}$ Ecnp678nk] e Tutte le mamme

[www.youtube.com/watch?v=qAQfRG_wgiA].

\section{Nel blu dipinto di blu}

Il 1958 rappresentò un anno di svolta proprio nel tempio della tradizione, Sanremo. Contro tutte le previsioni, vinse Domenico Modugno con Nel blu dipinto di blu

[www.youtube.com/watch? $\mathrm{v}=\mathrm{nD} 8 \mathrm{BryVB} 9 \mathrm{~d} 0$ ] anzi trionfò, col pubblico in sala a cantare in coro il ritornello Volare oh ohh. Di lì a poco gli stessi cori risuonarono in tutto il mondo, anche negli stadi. Modugno costituiva una novità nel panorama musicale italiano: un cantautore (ma questa parola allora non si diceva, entrò nell'uso l'anno dopo), anzi un "cantattore" per la sua straordinaria capacità di calcare il palcoscenico. Si è detto che, con quelle braccia protese in alto, Modugno facesse allora "volare i sogni degli italiani": in fondo, il "miracolo economico" che iniziò quell'anno assomigliava molto a quel sogno, finalmente possibile, di volare nel blu. Sul piano strettamente musicale, quella canzone potrebbe apparirci non molto innovativa (e in effetti negli anni successivi Modugno "rientrò nei ranghi" della tradizione melodica), ma per ricredersi è sufficiente confrontarla con le due canzoni favorite quell'anno a Sanremo e sorprendentemente sconfitte: L'edera cantata da Nilla Pizzi [www.youtube.com/watch?v=hW7F3ULbIz4], e Fragole e cappellini da Claudio Villa [www.youtube.com/watch?v=dyt9a_CxHI4 ]

\section{Il rock'n' roll: la musica che "fece nascere" i giovani}

Una sfida ben più radicale alla tradizione melodica venne però dalla penetrazione del rock and roll (o rock'n'roll), che era molto più di una nuova musica da ballo: era la prima moda giovanile, che si diffuse dall'America in tutto il mondo. Non è eccessivo considerare quel processo un mutamento antropologico senza precedenti, ovvero la nascita dei giovani come soggetti diversi e separati dal mondo degli adulti, con gusti e mode loro: nella musica, ma anche nel vestiario, nel look, nel linguaggio, nei modi e nei luoghi di aggregazione. Gusti e mode significano anche, e soprattutto, modelli di consumo, per cui si può dire che i giovani nacquero come nuovo e specifico segmento di mercato, agli albori della diffusione dei consumi di massa. Tutto questo non esisteva fino agli anni 
Cinquanta: si era considerati bambini, poi di colpo adulti, passando direttamente dai calzoni corti a giacca e cravatta.

\section{Il boom discografico americano}

Il rock'n'roll era iniziato nel 1955 sulle note di Rock around the clock, cantata da Bill Haley[www.youtube.com/watch?v=ZgdufzXvjqw]. Lo stile di Haley, che urlava le canzoni, era adattissimo ai juke-box, diventati di moda proprio allora e piazzati in luoghi affollati e chiassosi. "Urlatori" furono chiamati in Italia i giovani cantanti che seguivano il rock'n'roll. Ben più di Bill Haley, però, il "profeta" della nuova musica fu Elvis Presley, l'idolo musicale più amato di tutti i tempi, i cui dischi iniziarono a penetrare in Italia proprio nel 1958, distribuiti dal colosso della discografia americana Rca (che pose fine così al monopolio discografico della Cetra). Quell'anno i dischi 45 giri soppiantarono definitivamente i vecchi 78 giri. I dischi venduti dai 3 milioni del 1951 e 9 milioni nel '56, schizzarono a quasi 17 milioni. In America il rock non fu una rivoluzione solo musicale: i giovani che ne erano protagonisti apparivano trasgressivi in tutto, come i Teddy Boys e le altre bande giovanili nate allora e immortalate da divi del cinema come Marlon Brando in "Il selvaggio" (1953) e James Dean in "Gioventù bruciata" (Rebels without a cause, 1955).

\section{Mina e Celentano prima di "Mina\&Celentano"}

In Italia la rivoluzione del rock venne molto annacquata. Tra i numerosi "urlatori" nostrani, tutti molto giovani, due comparvero per la prima volta in TV nel 1959, in una popolarissima trasmissione televisiva condotta da Mario Riva, il Musichiere: Mina e Adriano Celentano, che amava presentarsi come 1'Elvis Presley italiano. Mina reinterpretò Nessuno, una canzone melodica portata quell'anno a Sanremo da Wilma De Angelis e Betty Curtis

[www.youtube.com/watch?v=MIGr-B1Ntvc]: lo fece però "alla sua maniera", con una versione tra rock e swing, sincopata, vorticosa, straordinariamente energica, che dà pienamente il senso della distanza tra urlatori e melodici [www.youtube.com/watch?v=ds8ItyxLLIM]. Già l'anno dopo, Mina abbandonò lo stile da urlatrice rock e divenne quasi subito un'interprete "classica": la più apprezzata a livello internazionale, la più grande di sempre per l'estensione e la versatilità di una voce che le consentiva di cantare brani di ogni genere, sempre fornendo esecuzioni superlative, come già nel 1960 con il capolavoro del cantautore Gino Paoli Il cielo in una stanza [www.youtube.com/watch?v=b6s_kjlhP5U] Adriano Celentano si presentò nel 1961 a Sanremo con Ventiquattromila baci [www.youtube.com/watch? $\mathrm{v}=\mathrm{KbWpGBV} 4 \mathrm{KlA}$ ], con un effetto dirompente: la canzone dissacrava l'amore riducendolo a un ritmo tayloristico di mille baci all'ora («Niente bugie meravigliose / frasi d'amore appassionate / ma solo baci chiedo a te / ye ye ye ye ye ye ye ye! »), e Celentano la eseguiva con voce rabbiosa, dimenandosi come mai si era visto, addirittura voltando le spalle al pubblico!

Le case discografiche e i giornalisti di costume sfruttarono la novità per lanciare la competizione tra urlatori e melodici, che almeno in parte era anche una competizione tra giovani e adulti, come accadde nel 1962 al Cantagiro, il nuovo festival itinerante nato quell'anno. Anche Celentano, come Mina, abbandonò molto presto l'immagine dell'urlatore, ed entrambi intrapresero carriere artistiche di grande successo ed eccezionalmente durature. Ai loro esordi, comunque, Mina e Celentano inaugurarono un fenomeno che sarebbe durato per tutti gli anni Sessanta: l'irruzione dei giovanissimi come protagonisti della musica.

\section{Un mondo di giovani e giovanissimi}

Nei primi anni Sessanta aumentarono moltissimo la produzione e il consumo di musica, e sia i consumatori di musica sia i cantanti furono soprattutto giovani e giovanissimi, in particolare $\mathrm{i}$ teenager. 
Quando ci riferiamo al consumo di musica, dobbiamo tenere presente che in quegli anni il suo veicolo principale era il disco in vinile (gli altri erano i pochi canali della radio, solo pubblica, e quelli della televisione, allora disponibile però solo per una minoranza di famiglie italiane). Le vendite di dischi passarono dai 18 milioni di 45 giri del 1959 ai 44 milioni del '69. Già nel 1960 un'inchiesta accertò che il 40\% degli acquirenti di dischi aveva meno di 20 anni, e che per loro era normale acquistare mediamente un 45 giri alla settimana. Ancora una volta, si trattava di un fenomeno già accaduto negli Stati Uniti alcuni anni prima. Le case discografiche furono le prime a rendersene conto e a fare lo stesso anche in Italia, cioè a "inventare" i giovani come specifica e molto redditizia fascia di consumatori.

Ovviamente la dilatazione del mercato riguardò anche gli adulti, che non avevano affatto smesso di ascoltare canzoni, di canticchiarle o fischiettarle e di ballarle. A quell'epoca, un disco di successo vendeva un milione di copie (il paragone col presente sarebbe falsato dal crollo del mercato discografico dovuto a internet; per fornire comunque un termine di paragone, oggi è considerato un successo un disco che venda 30.000 copie).

Per portare in giro la musica, e magari ascoltarla in spiaggia o in festini improvvisati, nacque allora il mangiadischi, un giradischi portatile a transistor per i 45 giri, per alcuni anni molto in voga tra i giovani. Nel 1959 sui settimanali iniziò la Hit parade (la classifica dei 10 dischi più venduti, nata in America molti anni prima), e nel 1967 ne iniziò la versione radiofonica, molto ascoltata dai teenager.

\section{Demografia, giovani e musica}

Apriamo una parentesi su alcuni dati demografici fondamentali per capire il rapporto tra musica, mercato discografico e giovani in quegli anni; ma forse anche per capire il ruolo che i giovani ebbero nelle vicende sociali e politiche in quel decennio e nel successivo.

Negli anni Cinquanta, non solo in Italia ma in gran parte dell'Occidente, si era interrotto il rallentamento della crescita demografica, per effetto del cosiddetto "baby boom", una temporanea ripresa della natalità che in Italia aveva toccato il suo culmine nel 1964, quando la fertilità era risalita all'alto livello medio di 2,7 figli per donna. Già nei secondi anni Sessanta quel "baby boom" cessò, e nei Settanta si arrivò alla cosiddetta "crescita 0": un equilibrio al ribasso tra mortalità e natalità, con mediamente due figli per donna (o per coppia), che garantiva il ricambio generazione. Dagli anni Novanta, però, l'Italia è diventata uno dei due-tre paesi al mondo a più bassa natalità, arrivando negli ultimi anni al non invidiabile record di soli 1,2 figli per donna, con la conseguenza di un fortissimo invecchiamento della popolazione (anche per la durata media della vita sempre più lunga): insomma, una società di "culle vuote" e con pochissimi giovani.

Confrontiamo qui la situazione attuale e quella degli anni Sessanta, ad esempio del 1965.

Anno

Popolazione residente in Italia, in milioni

Percentuale della popolazione sotto i 20 anni

Percentuale della popolazione sopra i 60 anni

Percentuale della popolazione tra i 15 e i 25 anni

Popolazione tra i 15 e i 25 anni, in milioni (M)
1965

$51,7 \mathrm{M}$

$32,6 \%$

$14,8 \%$

$15,1 \%$

$7,8 \mathrm{M}$
2019

$17,9 \%$

$30,2 \%$

$9,6 \%$

$5,7 \mathrm{M}$

(da www.populationpyramid.net/italy)

Si può notare che i giovani tra i 15 e i 25 anni erano discretamente ( 2 milioni) più numerosi di quelli di oggi, nonostante la popolazione totale comprenda oggi 8 milioni più residenti in più. Non si può certo ricondurre tutto a dati meramente quantitativi, ma neppure sottovalutare il peso demografico che i giovani ebbero in quegli anni, come parte preponderante del mercato discografico, ma ovviamente anche nei processi ed eventi più rilevanti della vita collettiva di quegli anni. $\mathrm{Ci}$ 
riferiamo agli studenti che affluirono nella scuola riformata nei primi anni Sessanta e poi nelle università, dove si sviluppò la contestazione del Sessantotto; agli operai, in gran parte giovani, che combatterono le lotte sindacali dall" "autunno caldo" del 1969 fino alla metà dei Settanta; alle donne, quasi sempre giovani, dei collettivi femministi e dei movimenti femminili protagonisti di importantissime battaglie nel campo dei diritti civili. Quelle diverse forme di sensibilizzazione sociale e politica, nella direzione dell" "impegno" e della "azione collettiva", emersero però verso la fine del decennio, contribuendo a cambiare il clima culturale, e quello musicale, negli anni Settanta.

\section{L'inizio dei Sessanta: la quiete prima della tempesta}

Per gran parte degli anni Sessanta, soprattutto nella prima metà del decennio, prevaleva invece un clima "leggero", all'insegna di un incontenibile ottimismo e di una spensierata vitalità. L'anno d'oro della musica italiana fu il 1964, quando vennero censiti in Italia 1.230 cantanti, 111 case discografiche, 770 dancing e night, 6.200 tra complessi e orchestrine: numeri incredibili! Fu in quegli anni che esplose la mania dei festival e dei concorsi canori, che si svolgevano a centinaia, nelle più remote località: tra il 1959 e il 1967 si tenne una media di 90 festival all'anno, con una punta di 119 nel 1965! I più noti furono il festival delle voci nuove di Castrocaro, il Cantagiro (dal 1962 al '70, una specie di "giro d'Italia" della canzone); il Festivalbar (che premiava i dischi più gettonati nei juke-box); il Disco per l'Estate (e dal 1957 era iniziato lo Zecchino d'oro, riservato ai bambini). I festival favorivano lo spirito di competizione tra "urlatori" e "melodici", costruito ad arte dalle case discografiche, dai rotocalchi e dalla TV all'inizio del decennio.

\section{La nascita del "divismo canoro"}

Sempre nei primi anni Sessanta le case discografiche imposero l'identificazione tra la canzone e il/la cantante che la lanciava, mentre fino agli anni Cinquanta la stessa canzone era interpretata indifferentemente da diversi cantanti. Questa personalizzazione dilatò sia il fenomeno del divismo canoro, sia la moda dei teen-idols, i cantanti-ragazzini coetanei del loro pubblico. Mentre negli anni Cinquanta i cantanti celebri erano per lo più trentenni o quarantenni, ora l'età si abbassò di colpo. Mina e Celentano avevano fatto scuola: dopo di loro, nel 1962 emersero Rita Pavone e Gianni Morandi, entrambi lanciati a 17 anni nella trasmissione televisiva "Alta pressione", sul neonato secondo canale della Rai [www.youtube.com/watch?v=0jQukiAOXXM]. Oltre ad essere giovanissimi, la Pavone e Morandi avevano in comune un modo di cantare su un unico timbro, senza estensione, che si prestava perfettamente alla nuova tecnica discografica di incidere separatamente la base musicale e la voce. Erano dunque prodotti perfetti da lanciare sul mercato musicale. Rita Pavone [Datemi un martello in mix twist, vedi dopo, e La partita di pallone: www.youtube.com/watch?v=8YqUHVoIkRQ ] conobbe un successo strepitoso ma breve, al contrario di quello di Morandi. Oltre a loro due, ci furono molti altri cantanti-ragazzini di successo, come Little Tony (Riderà, Cuore matto), Remo Germani (Baci), Bobby Solo (Una lacrima sul viso, Una granita di limone), Dino (Gli occhi miei), Michele (Se mi vuoi lasciare), Massimo Ranieri (Rose rosse, Se bruciasse la città), Fausto Leali (A chi, Deborah); e donne che proponevano immagini femminili molto diverse: dalla "acqua e sapone" Giliola Cinquetti di Non ho l'età per amarti [www.youtube.com/watch?v=0bTv1-fb61U] (che trionfò a Sanremo e all'Eurofestival del 1964) alla sexi e maliziosa Caterine Spaak di Quelli della mia età; a protagoniste dalla seconda parte del decennio come l'indipendente Caterina Caselli di Nessuno mi può giudicare [www.youtube.com/watch?v=R8XAHNbyWIs] e la trasgressiva Patty Pravo di Ragazzo triste [vedi alla fine], La bambola e Qui e là, a Nada (Ma che freddo fa). Morandi e alcuni altri si improvvisarono anche protagonisti dei "musicarelli", film musicali di infimo livello girati in poche settimane, nei quali giovani cantanti di successo recitavano se stessi, come Morandi in In ginocchio da te [www.youtube.com/watch? $\mathrm{v}=\mathrm{VvWH}$-jiwp2k] Oltre ai "musicarelli" nacquero allora i "cinebox", antenati degli attuali videoclip.

La contrapposizione tra melodici e urlatori durò poco, sia perché la generazione dei cantanti degli anni Cinquanta uscì di colpo di scena, con poche eccezioni (la più rilevante, "il reuccio" Claudio 
Villa); sia perché ovviamente la canzone tradizionale continuava ad avere un pubblico appassionato, tra i meno giovani ma anche tra i giovani, e nuovi interpreti, come Ornella Vanoni e Milva (spesso paragonate a Mina: del vastissimo repertorio della prima, da ricordare almeno Senza fine, Domani è un altro giorno, Eternità; della seconda Little man, La filanda). Poi Orietta Berti (Io tu e le rose, Fin che la barca va), Iva Zanicchi (Non pensare e me, Come ti vorrei, Zingara), i già citati Massimo Ranieri e Giliola Cinquetti, Jimmy Fontana (Il mondo, La nostra favola), Nicola di Bari (Il mondo è grigio il mondo è blu, poi La prima cosa bella e Vagabondo) e molti altri. In definitiva, poi, il repertorio di molti cantanti comprendeva canzoni di diversi registri.

\section{Tormentoni estivi ante litteram}

Con il "miracolo economico" iniziò il turismo di massa, e con quello il filone delle "canzoni da spiaggia": lo inaugurò Legata a un granello di sabbia, la prima a superare il milione di dischi venduti, cantata nel 1961 da Nico Fidenco, che replicò l'anno dopo con Con te sulla spiaggia [vedi dopo in mix twist]. Poi lo specialista divenne Edoardo Vianello, con pezzi cadenzati e molto orecchiabili come Pinne fucile ed occhiali [www.youtube.com/watch?v=7cXbcoD698M ], Abbronzatissima, Il peperone.

Ad alimentare il genere vi era anche, come già ricordato, il concorso Disco per l'estate. Citiamo alcuni altri dei "tormentoni estivi" più celebri negli anni Sessanta: due successi clamorosi dei Los Marcellos Ferial, cioè nel 1962 Quando Calienta el sol (1962) e Sei diventata nera (1964) che stravinse la prima edizione del Disco per l'estate di Saint Vincent [www.youtube.com/watch?v=gSFm5DAafHE]; poi Luglio (1968, Riccardo Del Turco) [www.youtube.com/watch?v=UbBKYfVj334] e Ahi le Hawai (1969, Herbert Pagani) [www.youtube.com/watch?v=DDMSu3fwVXo] e nello stesso anno Domenica d'agosto di Bobby Solo. Nel genere si cimentarono anche Mina, che nel 1963 interpretò Stessa spiaggia stesso mare di Piero Focaccia [www.youtube.com/watch?v=24pBdjenmWs], e il cantautore Gino Paoli con Sapore di sale nel 1963 [www.youtube.com/watch? $\mathrm{v}=$ MoJOREXYq4], arrangiata dal maestro Ennio Morricone. Ad eccezione di quest'ultima, le "canzoni da spiaggia" non erano certo di eccelso valore, ma la loro facile orecchiabilità conferì alla musica degli anni Sessanta la sua inconfondibile atmosfera di superficiale spensieratezza e di gioiosa vitalità. A questo contribuiva anche il fatto che esse erano quasi sempre associate a nuovi balli giovanili, uno - o spesso più di uno - per ogni estate: limbo, calypso, cha cha cha, hully gully, yéyé, surf, shake ma soprattutto il twist [www.youtube.com/watch?v=EJAyjbXx15s]. Nato negli Stati Uniti nel 1960 [www.youtube.com/watch?v=MggQSspSGU8], esso segnò una svolta perché veniva ballato in gruppo, non in coppia. Non venne meno, però, lo spazio per il "lento", cheek to cheek, che accomunava giovanissimi e adulti, rimasti legati alle atmosfere dei night (come E la chiamano estate di Sergio Martino o Una rotonda sul mare di Fred Bongusto). Fu allora che i locali da ballo per i giovani, che dai Settanta si sarebbero chiamati discoteche, si differenziarono da quelli per gli adulti. Fino agli anni Cinquanta, adulti e giovani ascoltavano e ballavano la stessa musica negli stessi locali, dai sofisticati night alle popolari "balere" per il "liscio".

\section{Apertura all'estero: da Paul Anka alla Beat generation}

Tipico degli anni Sessanta fu anche il fatto che molti celebri cantanti stranieri venivano a cantare in Italia in italiano, attirati da un mercato musicale così vasto e recettivo, e dalla passione tutta italiana per i festival canori: così accadde a Neil Sedaka (Esagerata), Paul Anka (Diana, Ogni volta), Dalida (La danza di Zorba, Bang bang), Francoise Hardy (Quelli della mia età), Petula Clark (Ciao ciao), Silvie Vartan (Come un ragazzo), Gene Pitney (Amici miei), Rocky Roberts (Stasera mi butto), Antoine (La tramontana), George Moustaki (Lo straniero), e a molti altri. L'interscambio tra canzone italiana e internazionale era strettissimo e molto "disinvolto": molte canzoni erano copiate di sana pianta da grandi successi internazionali, con traduzioni chiamate cover.

A metà dei Sessanta iniziò la musica della beat generation, anch'essa proveniente dal mondo anglosassone, da esperienze molto diverse: i due grandi folksinger americani Bob Dylan e Joan 
Baez, e i "complessi" (così erano allora chiamati i gruppi musicali), a partire dai Beatles e dai Rolling Stones.

Nonostante l'enorme rilevanza del fenomeno Beatles per la musica leggera mondiale, all'inizio la loro penetrazione in Italia fu lenta e difficile[1], perché la barriera linguistica era allora un ostacolo molto più forte che nei decenni successivi. Non a caso, ebbero inizialmente un maggior successo $\mathrm{i}$ complessi stranieri (così come i solisti) che in quegli anni venivano a cantare in italiano, quali $\mathrm{i}$ Rockes (E la pioggia che va, Ma che colpa abbiamo noi), Mal dei Primitives (Tu sei bella come sei), i Motown (vedi dopo), e i complessi italiani, come gli Equipe 84 (29 settembre), i Nomadi (Come potete giudicar, Io vagabondo), i Ribelli (Pugni chiusi), I profeti (Gli occhi verdi dell'amore), i Giganti (Tema), i Camaleonti (Applausi, L'ora dell'amore), i Dik Dik (Sognando California, L'isola di Wight), i Pooh (Piccola Katy, Pensiero), i New Trolls (Signore, io sono Irish nel 1968, e Una miniera nel 1969: www.youtube.com/watch?v=V6HA35UtaVU ).

\section{L'essere giovani come bandiera identitaria}

In diversi casi, quei giovani che cantavano per i loro coetanei fecero dell'essere giovani il tema stesso delle loro canzoni, con un'orgogliosa affermazione di identità generazionale. Può essere interessante soffermarsi su alcuni esempi, molto diversi tra loro sia musicalmente che per i temi e le atmosfere.

Pino Donaggio (1941), prima di avere una grandissima carriera internazionale di compositore di colonne sonore di film, esordì ventenne come cantautore, e nel 1963 (due anni prima di comporre $I o$ che non vivo senza te, che vendette 80 milioni di dischi in tutto il mondo), si piazzò terzo a Sanremo con il twist Giovane giovane, che aveva tutte le caratteristiche di un inno a chi si affaccia con spensieratezza e ottimismo alla vita adulta. Ne proponiamo il video-ascolto in un tipico cine-box di

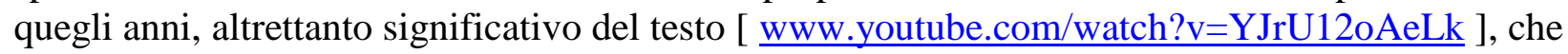
iniziava così:

«E' tanto breve la primavera / nessuno la ferma con sé I

Ma tu la porti nel tuo sorriso / sorriso che dedichi a me / [segue il ritornello:] Giovane giovane giovane / hai tutta una vita da vivere ancor /

Ridono ridono ridono / ti ridono gli occhi pensando all'amor /

Svegliati svegliati svegliati / milioni di baci aspettano te /

Spendili spendili spendili / tutti tutti con me ...».

Nel 1964 il grandissimo paroliere Mogol (che produsse testi per tutti i maggiori cantanti dei Sessanta, prima di costituire un sodalizio decennale con Lucio Battisti) scrisse una canzone che fu interpretata dalla giovanissima cantante-attrice belga Caterine Spaak, ed entrò anche nella colonna sonora del capolavoro cinematografico di Dino Risi Il sorpasso, nel quale la Spaak recitava con Vittorio Gassmam. Al ritmo del surf, un ballo estivo di quegli anni [www.youtube.com/watch?v=TfCBhqr8LYo], la canzone iniziava con il ritornello:

« Noi siamo i giovani, i giovani, più giovani / siamo l'esercito, l'esercito del surf /[e proseguiva] Ma che cosa c'è / balla insieme a me / E vedrai che poi / ti passerà /

Mi vuoi dir perché non sorridi più / Balla insieme a me (bis) / poi ti passerà...».

\section{Il Piper}

Nel 1966 l'allora ventunenne Gianni Pettenati incise Bandiera Gialla, cover dell'inglese The pied piper. La canzone, destinata a diventare una evergreen, era allora associata al locale romano Piper, il nuovo luogo di aggregazione dei giovanissimi della beat generation. Soprattutto, Bandiera Gialla 
fu il titolo di un popolarissimo programma radiofonico di canzoni trasmesso da Radio 2 tra il 1965 e il '70, condotto da Renzo Arbore e Gianni Boncompagni, che iniziava con questa provocazione: «A tutti i maggiori degli anni 18: questo programma è rigorosamente riservato ai giovanissimi, ripeto ai giovanissimi; tutti gli altri sono quindi pregati di spegnere la radio o di sintonizzarsi su altra stazione». Il video, con il cantante circondato da giovani che ballano la sua canzone, appare un

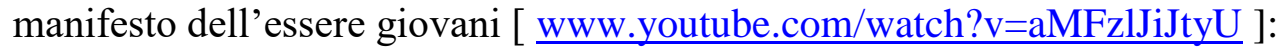

«Sì, questa sera è festa grande / noi scendiamo in pista subito /

E se vuoi divertirti vieni qui / ti terremo tra di noi, e ballerai /

Finché vedrai sventolar bandiera gialla / tu saprai che qui si balla, ed il tempo volerà /

Saprai, quando c'è bandiera gialla / che la gioventù è bella, e il tuo cuore batterà /

Sai, quelli che non ci voglion bene / è perché non si ricordano /

Di essere stati ragazzi giovani / e di avere avuto già la nostra età...»

In un video del 1967 all'interno del Piper, il locale di moda dei giovani beat di Roma, Nicoletta Strambelli (1948), in arte Patty Pravo, prima di cantare si presentò così:

«Io sono Patty Pravo. Canto qui al Piper... io penso che questo sia un po' il mondo di noi giovani. Prima di cantare, io venivo qui a ballare. Noi giovani siamo molto uniti tra di noi, perché con l'altra generazione non ci troviamo molto bene, e poi non sanno ballare ».

Subito dopo iniziò a ballare e a cantare il suo grande successo, Ragazzo triste, sopra una pista da ballo gremita di adolescenti [ $\underline{w w w}$.youtube.com/watch?v=LgOhkh6dOrg ]:

«Ragazzo triste come me ah ah / che sogni sempre come me ah ah /

Non c'è nessuno che ti aspetta mai / perché non sanno come sei /

Ragazzo triste sono uguale a te / a volte piango e non so perché I

Tanti son soli come me e te / ma un giorno spero cambierà /

Nessuno può stare solo / non deve stare solo / quando si è giovani così /

Dobbiamo stare insieme / parlare tra di noi / scoprire insieme il mondo che ci ospiterà...»

\section{Il gruppo di Liverpool (che non sono i Beatles)}

Proponiamo un ultimo esempio, dello stesso 1967, quando in un programma televisivo Gianni Morandi presentava il gruppo "venuto da Liverpool" (come i Beatles, e con lo stesso taglio dei capelli) dei Motown, che cantavano (con un vistoso playback, sic!) il loro successo italiano, Prendi la chitarra e vai [www.youtube.com/watch?v=JhvF1VLb6YA ]:

«Prendi la chitarra e vai / per il mondo dove vuoi /

sono tante le strade ma poi / quella giusta troverai /

canta canta $i$ sogni tuoi / prendi la chitarra e vai /

Per il mondo tu vedrai / gente che non ride più /

Che non sa cercare ormai / ciò che stai cercando tu /

Il denaro è bello ma / conta più la libertà /

Prendi la chitarra e vai / se ti fermi invecchierai /

Oggi il mondo si burla di te / forse un giorno ascolterà /

Canta canta i sogni tuoi / prendi la chitarra e vai /

Sui giornali leggerai / che la pace si farà / (Nb: forti applausi del pubblico)

Basta star con questi qua / e far fuori quelli là /

Ma per quello che ne so / basterebbe dirgli no /

Prendi la chitarra e vai / tu sei giovane perciò / 
Non vuoi male a nessuno perché / senti tutti uguali a te /

Canta canta i sogni tuoi / prendi la chitarra e vai. »

In questo caso, abbiamo riprodotto integralmente l'esecuzione del testo, per lasciare riconoscere gli espliciti riferimenti sociali e politici (al denaro e alla guerra) e l'atmosfera vagamente da "figli dei fiori” tipica degli anni a ridosso del Sessantotto.

Un mondo a parte: $i$ cantautori e la "canzone impegnata"

Tutt'altro mondo era quello dei cantautori degli anni Sessanta, appartati e lontani dal gusto del grande pubblico. Intellettuali prima che cantanti, si ispiravano al jazz, alla musica francese e alla filosofia esistenzialista; erano alternativi nei valori e negli stili; nelle loro canzoni denunciavano il conformismo e l'ipocrisia della società borghese, oltre a toccare temi più intimi, come la difficoltà della relazione e il "male di vivere". Adottavano la lingua parlata del quotidiano, disadorna, spesso cruda nei termini; per la prima volta, in alcuni dei loro testi abbandonavano la rima e altre convenzioni poetiche consolidate: insomma, le loro creazioni erano l'opposto delle superficiali canzonette all'italiana.

\section{Da Genova a Milano, da Milano all'Emilia}

I primi cantautori facevano parte della cosiddetta "scuola genovese": Gino Paoli (Sassi, Il cielo in una stanza: www.youtube.com/watch? $\mathrm{v}=\mathrm{jU} 43 \mathrm{Dc} 9 \mathrm{KZbE}$ ), Luigi Tenco (Io sono uno 1966, Ciao amore ciao, 1967: www.youtube.com/watch?v=MbFYwkgu0L4), Fabrizio De André (Bocca di rosa, 1967: www.youtube.com/watch?v=JrFjFOjxYyA), Bruno Lauzi, oltre al triestino Sergio Endrigo (Teresa, 1966: www.youtube.com/watch?v=3Zr286oLrB4).

Le loro opere, di grande spessore poetico, trovarono inizialmente poco ascolto presso il grande pubblico: Paoli e De André divennero famosi per l'interpretazione che Mina diede ad alcune delle loro prime canzoni (Il cielo in una stanza del primo, qui riprodotta all'inizio, e La canzone di Marinella del secondo), mentre l'incomprensione del pubblico contribuì alla tragica fine di Tenco, a Sanremo nel 1967.

Da un altro contesto, l'ambiente del teatro politico milanese, emersero invece Enzo Jannacci e Giorgio Gaber, che dopo avere esordito in coppia come cantanti rock [vedi Una fetta di limone, nel mix twist, dopo ] proseguirono separatamente, negli anni Settanta, ciascuno con uno stile originalissimo, ma entrambi nella direzione di canzoni di significato politico e sociale [Enzo Jannacci, L'Armando (1964): www.youtube.com/watch?v=9851133DMfs; Giorgio Gaber, La libertà (1972): www.youtube.com/watch?v=j3vowbyQBiQ].

Le originali ballate folk del cantautore modenese Francesco Guccini erano poesie o racconti in musica, anch'esse spesso politicamente impegnate, come Auschwitz e Dio è morto $[w w w . y o u t u b e . c o m / w a t c h ? v=K h J 2 e e L B q A c]$, inizialmente affidate all'esecuzione dei due complessi modenesi e reggiani con cui collaborò, cioè gli Equipe '84 e i Nomadi. Guccini creò nel 1972 La locomotiva, che divenne la canzone-simbolo dell'impegno politico del "lungo Sessantotto", assieme a poche altre, quali Signore, io sono Irish e La miniera dei New Trolls (1969, vedi sopra), C'era un ragazzo che come me amava i Beatles e i Rolling Stones, di Gianni Morandi (1966, e in quello stesso anno Contessa di Paolo Pietrangeli, appartenente però alla cerchia della musica militante, distante dai circuiti commerciali).

Si può dire che l'influenza del nuovo clima del Sessantotto si manifestò negli anni Settanta, in due direzioni: da una parte, finirono il "giovanilismo" dei teen-idols, l'ubriacatura dei concorsi canori, la spensierata e superficiale atmosfera delle "canzoni da spiaggia"; dall'altra parte, i cantautori, rimasti ai margini negli anni Sessanta, presero invece il centro della scena musicale, con testi e 
composizioni di ben altro spessore: sia alcuni di quelli sopra citati, sia una "seconda generazione" di cantautori, composta da Battisti, Dalla, De Gregori, Venditti, Baglioni, Fossati, Battiato e vari altri. Ma dei cantautori, e di quel decennio, si parla in un altro contributo.[2]

\section{Bibliografia e sitografia}

- Gianni Borgna, Storia della canzone italiana, Roma-Bari, Laterza 1985

- Felice Liperi, Storia della canzone italiana, Roma, Rai-Eri 1999

- Edmondo Berselli, Canzoni. Storie dell'Italia leggera, Bologna, Il Mulino 1999

- Guido Crainz, Storia del miracolo italiano, Roma, Donzelli 2003

- Enzo Gentile, Legata a un granello di sabbia. Storie e amori, costume e società nelle canzoni italiane dell'estate, Milano, Melampo Editore 2005

- Leonardo Colombati (a cura di), La canzone italiana 1861-2011, Milano, Mondadori 2011

- Alberto De Bernardi, Un paese in bilico, Roma-Bari, Laterza 2014

- Guido Crainz, Storia della repubblica, Roma, Donzelli 2016

- Cesare Grazioli, L'Italia in musica, sul sito dell'Università di Lione, all'indirizzo:

- http://cle.ens-lyon.fr/italien/l-italia-in-musica-indice-162864.kjsp?RH=CDL_ITA110500

Note:

[1] Si veda: Ferdinando Fasce, The Beatles in Italy. Per una ricerca sulla storia della popular music e i lunghi anni Sessanta, Novecento.org, n. 12, agosto 2019. http://www.novecento.org/lacolonna-sonora-di-una-generazione/the-beatles-in-italy-per-una-ricerca-sulla-storia-della-popularmusic-e-i-lunghi-anni-sessanta-4647/

[2] Si veda http://www.novecento.org/?p=4684 